\title{
Structural Validity of the School Aspirations Questionnaire (SAQ)
}

\author{
Cristiano Mauro Assis Gomes* \& Evgjeni Gjikuria \\ Universidade Federal de Minas Gerais, Belo Horizonte, MG, Brasil
}

\begin{abstract}
The self-determination theory claims that three basic needs (autonomy, relatedness, and competency) guide the human motivation. This theory serves as background for many psychological instruments. However, no one differentiates the students' occurrence perceptions and students' values about the basic needs. The School Aspirations Questionnaire has this goal, but its structural validity was not investigated yet. The present study evaluated the structural validity of the questionnaire. The sample was composed by 716 middle and high school students from a private school in Belo Horizonte, Brazil. A model was tested. This model possessed eight correlated latent variables representing, in terms of values and occurrence perceptions, the three basic needs and the immediate pleasure domain. The model showed good data fit, permitting the conclusion that the questionnaire is capable of distinguishing in the school context the values and the occurrence perceptions about the basic needs, as well the immediate pleasure domain.
\end{abstract}

KEYWORDS: validity, psychological instrument, self-determination theory, perceptions, values

\section{Validade Estrutural do Questionário de Aspirações Escolares}

\begin{abstract}
RESUMO - A teoria da autodeterminação afirma que três necessidades básicas (autonomia, vínculo e competência) norteiam a motivação humana. Muitos instrumentos psicológicos têm como base esta teoria. No entanto, nenhum distingue as percepções de ocorrência das necessidades básicas, e o quanto estas necessidades são valorizadas. O Questionário de Aspirações Escolares foi criado com esse objetivo, mas carece de qualquer análise de sua validade estrutural. O presente estudo pretende realizar esta análise. Os dados analisados provêm de 716 estudantes, ensino fundamental II e médio, de uma escola privada de Belo Horizonte, Minas Gerais. Foi testado um modelo com oito variáveis latentes correlacionadas representando as necessidades básicas e o domínio do prazer imediato, em termos de percepção e valores. O modelo apresentou bom ajuste aos dados, possibilitando concluir que o questionário é capaz de distinguir, no contexto escolar, os valores e as percepções sobre as necessidades básicas e o domínio do prazer imediato
\end{abstract}

PALAVRAS-CHAVE: validade, instrumento psicológico, teoria da autodeterminação, percepções, valores

\section{SELF-DETERMINATION THEORY}

We should suppose that any teacher, or at least the majority of them, aspire their students to have their own act of learning as one of the most important aspects of continuance of studying and learning. Discovering the beauty involved in the interaction with an object of knowledge is perhaps the most powerful motive for the longstanding desire of learning, for overcoming challenges and for achieving higher levels of mastering (Early, Rogge, \& Deci, 2014).
Of course, many reasons can impulse students to learn. The shame or the punishment in getting a bad grade, or the perspective of getting a better life through education are some examples of the many possible reasons. However, despite the diversity of motives, perhaps the most impactful drive to learn is the intrinsic motive of learning for the own act in itself (Hagger \& Chatzisarantis, 2016).

The self-determination theory has the merit of providing concepts capable of articulating the relations between

*Email: cristianomaurogomes@gmail.com 
intrinsic and extrinsic motives of learning, as well as proposing strategies for teachers which intend to promote goals and actions more autonomous and self-regulated to their students (Hagger \& Chatzisarantis, 2016). The theory assumes the existence of a proactive tendency in every human being to engage in social and physical challenges, as well as assimilate the values and cultural practices present in the environment, premising that people are inherently curious, interested, and possess a natural love for learning and a desire for acquiring knowledge, cultural habits and values that surround them (Chen et al., 2015). Apart from this, the theory assumes that intrinsic motivation is sustained by the satisfaction of three basic needs - autonomy, competency and relatedness - which seems to be supported by evidence (Hagger \& Chatzisarantis, 2016).

The basic needs of autonomy, competence and relatedness are grasped as fundamental motives of human being, present in all cultures. The necessity of autonomy is described as the person's propensity to evolve himself and his own inner forces through an active, authentic, self-endorsed and personal style (Deci \& Ryan, 2016). The necessity of mastering the objects of knowledge and becoming competent, overcoming the challenges, characterizes the need for competence. In turn, the necessity of relatedness is defined as the propensity to search meaningful relations with other human beings, assimilating the culture, values and sociocultural meanings (Chen et al., 2015).

There is the acknowledgement that all activities are neither interesting nor intrinsic motivators. In these cases, the theory argues that an intrinsic motivation is not evident and, because of that, people need other incentives or reasons to act (Deci \& Ryan, 2016). The extrinsic motivation helps to explain this context, because it describes all the behaviors which the finality of the actions encounter separated of the activity in itself. Extrinsic motivation occurs when the reward of an activity is extrinsic to the task in itself. The selfdetermination theory defines that the gap between intrinsic motivation and extrinsic motivation is not binary, and, hence, there is a continuum between them (Deci \& Ryan, 2016). There are classes of extrinsic motivations and some of them are so close to intrinsic motivation. These are related to an internal locus of control and personal causality, tending to generate more satisfaction, interest, engagement, better achievement and adjustment in life (Black \& Deci, 2000).

About the practical implications of the self-determination theory, there are a great number of health and educational behavior interventions which focus on the support of the self-determination's basic needs, fostering the process of internalization into more autonomous forms of selfregulation. Silva, Marques and Teixeira (2014), as well Hagger and Chatzisarantis (2016) provide an extensive review of these interventions, showing that it should provoke a direct effect on motivation, engagement, learning and psychological well-being, reinforcing the claim that self-determination theory possesses favorable evidence in different cultures that the support on autonomy, competency and relatedness is associated to better engagement and achievement, as well as quality of life.

\section{QUESTION OF STUDY}

There are many questionnaires that intend to measure the three fundamental needs of autonomy, competency and relatedness, along with measuring the continuum between intrinsic and extrinsic motivations. For example, the General Causality Orientations Scale (GCOS) measures three motivational orientations: autonomy orientation, controlled orientation, and impersonal orientation (Deci \& Ryan, 1985); the Learning Climate Questionnaire (LCQ) measures the instructors' autonomy support perceived by the students (Black \& Deci, 2000); the Academic SelfRegulation Questionnaire (SRQ-A) measures external, introjected, identified and intrinsic regulations (Ryan \& Connell, 1989); the Basic Psychological Needs Scale (BPNS) measures the satisfaction in the three basic needs, competency, autonomy and relatedness (Deci \& Ryan, 2000); the Reading Motivation Scale measures the extrinsic controlled motivation for reading, the extrinsic autonomous motivation for reading, the intrinsic motivation for read and the demotivation for reading (Gomes \& Boruchovitch, 2015). Maybe the most recent is the Basic Psychological Need Satisfaction and Frustration Scale (BPNSFS), a 24-items questionnaire that assesses autonomy, relatedness, and competency, in terms of satisfaction or frustration of these needs. Chen et al. (2015) reported the construction and the cross-cultural validation of the BPNSFS, applying invariance confirmatory factor analysis of items in samples of university students from USA, Belgium, China and Peru.

However, until now, none of them measured the aspirations of people in relation with the needs of autonomy, competency and relatedness, discriminating the persons' perceptions for the occurrence of these needs, as well as the value which people give to each of them. Aiming to cover this gap, the first author of this manuscript created a self-report instrument that intends to do that, for middle school and high school students, which have to inform their perceptions about the occurrence of behaviors related to autonomy, competency and relatedness in the school context. Conjointly to that, they have to answer how much they value each of these behaviors of autonomy, competency and relatedness. Statements were created in the questionnaire that intend to measure a fourth aspect, beyond the three fundamental needs, which we consider important in the school context. This aspect is the evolvement of the students in doing the school activities ruled by the 
immediate pleasure. The theoretical rationale that sustains the immediate pleasure construct comes from the literature of hedonic pleasure and eudemonic pleasure. Walker, Winn and Lutjens (2012) provide a good example that distinguish these two constructs, informing that "someone may play the piano because it is fun and feels good in the moment (hedonic enjoyment) or as a means of developing their musical talent and pursuing their musical and/or creative potential (eudaimonic happiness)." (Walker et al., 2012, p. 2). Despite of hedonic pleasure should be empowering in certain experiences and contexts, this kind of motivational drive is limited and should bring problems to students, principally in the context of school learning, where the immediate pleasure is not the rule, but the exception. As Walter et al. (2012) explain, the problem with immediate pleasure is that "experiencing hedonic pleasure is often temporary and not sufficient to bring about a sense of longterm fulfillment" (Walter et al., 2012, p. 2).

This instrument, whose name is School Aspirations Questionnaire $(S A Q)$, possesses 31 statements which describe behaviors about autonomy, competency, relatedness, and immediate pleasure. The first author of this manuscript did not create the 31 statements through the previous items from the existent instruments, since any of them distinguish the values and the occurrence perceptions about the basic needs. Unfortunately, the School Aspirations Questionnaire should not be directly compared with the previous instruments. It is a consequence of its originality, since the existent instruments assess the satisfaction or the frustration of the basic needs. On the contrary, the School Aspirations Questionnaire is interested in assesses how people perceive the frequency of autonomy, competency and relatedness, in the school context, as well how they value these needs.

There is some evidence about the content validity of the SAQ. The 31 statements were evaluated through a content validity analysis. A referee evaluated if the statements corresponded to their target construct, classifying if the statement pertained to autonomy, competence, relatedness, or immediate pleasure. The statements which were not correctly related to their target by the referee were permitted to be associated to two constructs. Just a couple of statements have this condition. For example, statement 28 is related to autonomy and competency (see Table 1 about the relationships between the statements and their constructs).

About the structure of the SAQ, the respondent has to inform his perception about the occurrence of these 31 statements in his school life, as well the value he gives to each statement. To do that, he has to mark one option in a Likert scale of five points, for each statement, from indicating if the behavior associated with the statement does not occur in his school life (point 1 of the scale) until the point of scale that shows if it occurs totally in his school life (point 5 of the scale). At the same time, he has to use the same scale of five points to indicate how much he values the behavior associated with that statement. In other words, the student must choose one point of the scale of five points, which informs if he does not value the behavior (point 1 of the scale) up to the point of him totally valuing the behavior (point 5 of the scale) that is associated with the statement. Some details of the SAQ's structure which organizes the statements and the scale should be inspected in the section method.

Because each statement is evaluated twice by the student, one evaluation for its occurrence and other for its value, each statement generates two items. So, the questionnaire has 62 items, 31 items for the perception of the students about the occurrence of the behaviors of autonomy, competency, relatedness, and immediate pleasure in the school context, and 31 items for the students' values about those behaviors.

We present one example of statement for competence, autonomy, relatedness and immediate pleasure. Remembering that each statement serves for one item of value and for one item of perception. An example of autonomy statement is: "I take the responsibility for my choices"; An example of competence statement is: "I aim at learning and understanding the school subjects"; An example of relatedness statement is: "I see school as a place to make friends"; And an example of immediate pleasure statement is: "I only do quality activities that give me immediate pleasure."

As we have informed, an important difference of the SAQ in relation to other instruments that assess the basic needs (BPNS and BPNSFS) is that those instruments focus on assessing satisfaction or frustration of these needs. Instead of that, the SAQ evaluates the students' occurrence perceptions and values about these needs. That difference is subtle but relevant, since SAQ assesses how the students perceive behaviors of autonomy, competency and relatedness in their personal school life, in terms of frequency and value. In some cases, these perceptions should be well related to how they are satisfied, but it is not a rule or a condition. It is possible, for example, that students perceive behaviors of autonomy as little frequent but, at the same time, they should be satisfied in terms of that need. The same is true for the values. In some cases, values and satisfaction should be close and well connected, but it is not a rule or a condition.

The SAQ is originally written in Brazilian Portuguese language, and has not been tested in its structural validity. So, we test a model of eight correlated factors, which are the students': (1) occurrence perception of competence, (2) competence value, (3) occurrence perception of relatedness, (4) relatedness value, (5) occurrence perception of autonomy, (6) autonomy value, (7) occurrence perception of immediate pleasure, and (8) immediate pleasure value. We permit cross-loadings among the factors and its nontarget items, as suggested by Marsh, Morin, Parker and Kaur (2014), applying the exploratory structural equation modeling (ESEM). Marsh and colleagues (Marsh et al., 2014) has shown that the exploratory structural equation modeling is usually a better approach than the confirmatory 
factor analysis, in the case of questionnaires which intend to measure two or more factors, because confirmatory factor analysis impedes cross-loadings among factors and nontarget items, provoking worse model data fit and inflating the factor correlations. On the other side, exploratory structural equation model corrects these two problems.
Aiming at facilitating the communication, we will describe the eight factors using only a synthetic name and not its complete name. For example, instead of utilizing the complete name of the student's occurrence perception of autonomy factor we will only describe it as autonomy perception or autonomy perception factor.

\section{METHOD}

\section{Participants}

The participants of the study compounded a convenience sample of 716 students from a private school in Belo Horizonte, Minas Gerais State, Brazil. They were middle school students - sixth to ninth grades ( $59 \%$ of the sample) or high school students - tenth to twelfth grades $(41 \%$ of the sample), and possessed a main age of 13.75 years ( 2.11 years of standard deviation). The majority was female (53\%), presenting an equilibrium among the grades (sixth grade $=$ $13.1 \%$; seventh grade $=15.3 \%$; eight grade $=13.8 \%$; ninth grade $=16.2 \%$; tenth grade $=13.5 \%$; eleventh grade $=$ $13.9 \%$; twelfth grade $=13.5 \%$ ).

\section{The School Aspirations Questionnaire (SAQ)}

The first author of this paper have created the School Aspirations Questionnaire (SAQ). This instrument intends to measure eight latent variables: (1) competence perception, (2) competence value, (3) relatedness perception, (4) relatedness value, (5) autonomy perception, (6) autonomy value, (7) immediate pleasure perception, and (8) immediate pleasure value. All these latent variables pertain to the context of the students' life in middle school and high school.

The SAQ possesses 31 statements and assesses the construct of competency, autonomy, relatedness, and immediate pleasure, evolving the students' occurrence perceptions and values about these constructs. So, each statement possesses two items, one for value and other for occurrence perception. There are 31 statements, generating 62 items in total, 31 items for values and 31 items for occurrence perception. The questionnaire does not possess any time constraint to be answered and it is a self-report instrument in Brazilian Portuguese language.

The student has to choose one point from a Likert fivepoint scale for each statement of the questionnaire. This scale is used both for measuring the student's perception about the occurrence of the statement in his school life, as the scale is used for measuring the student's value about that statement. The five points of the scale are: (1) not even a little, (2) somewhat, (3) more or less, (4) much, (5) totally. So, for example, if the student thinks that the behavior underlying in a statement is not even a little existent in his life, he must mark the point 1 of the scale. It is his perception of occurrence about that statement in his life. If he values much that behavior, he needs to mark the point 4 of the scale, now indicating his value about that behavior. Hence, he would mark the point 1 of the scale for the occurrence perception of the statement in his life and he would mark the point 4 of the scale for his value about that statement.

The questionnaire has a structure that permits the students to correctly answer their response about the value and the perception of occurrence for each statement, as should be seen in the following example (figure 1):

\begin{tabular}{|lcc|}
\hline Statement & Occurrence & Value \\
\hline $\begin{array}{l}\text { I take the responsibility } \\
\text { for my choices. }\end{array}$ & $(\quad)$ & $(\quad)$ \\
I choose what I consider \\
the best for me.
\end{tabular}$\left(\begin{array}{l}\text { ) } \\
\hline\end{array}\right.$

Figure 1. Questionnaire Structure

Table 1 shows the statements and their theoretically target construct. In a few cases a statement is related to two constructs. The statements of autonomy describe behaviors of self-accountability, self-control, personal engagement and internal locus of control. The statements of immediate pleasure describe behaviors which are regulated and controlled by pleasure and its immediacy. The statements of competence represent behaviors of intrinsic motivation in learning, in addition to goals that obtain a good academic achievement and grades. So, these statements are not the representative of a unique polarity in the continuum between intrinsic motivation and extrinsic motivation. The statements of relatedness evolve principally behaviors of friendship and companionship among students and educators.

\section{Procedures}

Only psychologists or students of psychology, which were supervised by the first author of this manuscript, applied collectively the questionnaire in 2008. The application occurred in a private school of Belo Horizonte, Minas Gerais State, Brazil, after the consent of the school managers, teachers, students and their parents or legal guardians. All Ethics commitments were considered, and the research has had the approval of the Ethics Committee of the Federal University of Minas Gerais, Brazil, protocol 
Table 1. The 31 Statements of $S A Q$ and their Target Constructs

Autonomy (6 statements)

Statement 3: "I know when to expose and sustain from other people what I think is important for my life and what I want for me."

Statement 6: "I take the responsibility for my choices".

Statement 11: "I make the decisions for my life and do not leave this task exclusively to my parents, school, and so on.

Statement 15: "I have clarity about what I want".

Statement 18: "I choose what I consider the best for me".

Statement 28: "I define goals and plan my actions to achieve things which I want."

Immediate Pleasure (5 statements)

Statement 2: "The most part of my time I do things that I like".

Statement 13: "I choose people for school group tasks based on common interests, even if they are not my friends".

Statement 25: "I dedicate myself only on those subjects which please me."

Statement 26: "Even when I do not like an activity, I do it with excellence" (This item is the inverse).

Statement 30: I do with excellence only activities that give me an immediate pleasure".

Competency (15 statements)

Statement 1: "I approximate myself to the teachers which teach the subjects that I am interested in developing."

Statement 4: "I work with people which think differently from me."

Statement 5: "I search for activities which I am not so good at, aiming at working on my difficulties".

Statement 9: "I am capable of thinking in other ways to solve a problem when the first try does not work out".

Statement 12: "I have good grades in the school evaluations".

Statement 14: "I aim at learning and understanding the school subjects".

Statement 17: "I aim at being the best student of my class".

Statement 20: "I get upset when I receive a bad grade".

Statement 21: "I aim at improving my capacity in reading and interpretation".

Statement 24: "When I study, I have the goal of mastering the knowledge of something."

Statement 26: "Even when I do not like a certain school activity, I do it with excellence."

Statement 27: "I always choose a colleague to participate with me in the school group activities in relation to his or her competency."

Statement 28: "I create goals and plan my actions to achieve the things that I want".

Statement 29: "I search for additional school tasks when I need extra points in order to pass to the next school year."

Statement 31: "I have conversations with my teachers about subjects which are out of the school program, aiming at increasing my knowledge".

Relatedness (7 statements)

Statement 7: "I make friends through assignments or group meetings".

Statement 8: "I talk with all my classmates".

Statement 10: "I meet new people and make new friends at school".

Statement 16: "I see school as a place to make friends".

Statement 19: "I go out with my school friends to have fun".

Statement 22: "I prefer working alone than working in groups"; (this item is inverse).

Statement 23: "I have school friends which I should always count on".

number 456/07. The students assigned a free assent term $(<$ 18 years-old) or a free consent term ( $>=18$ years-old), as well their parents assigned the free consent term before the questionnaire application.

\section{Data Analysis}

We employed the exploratory structural equation modeling (ESEM) and the weighted least square of means and variance estimator (WLSMV), using the Mplus 7.0 statistical software (Muthén \& Muthén, 1998-2014). Because the SAQ has a Likert-type scale of 5 points, WLSMV treated the items' scores as categories with four thresholds. In function of ESEM needs a rotation estimation, we applied the target rotation, suggested by Marsh et al. (2014) as a good approach so as to maximize the confirmatory aspect in ESEM. This kind of rotation "provides a stronger a priori model, gives the researcher greater control in specifying the model, and facilitates the interpretation of the results" (Marsh et al., 2014, p. 90).
We run a model of eight correlated factors, permitting cross-loadings among the factors and its non-target items, as well correlating the pairs of items from the same statement. The cross-loadings in ESEM were modeled to be as close to zero as possible. The model syntax run is presented in Table 2.

We briefly explain the model syntax through an example (Table 2). The latent variable of relatedness value explains the variance of its target items, which are items av8, av10, av7, av16, av19, av22 and av23. The part of the model syntax which defines these relations is "Vrelatedness BY av8 av10 av7 av16 av19 av22 av23". Conjointly, the model syntax assumes that this factor loads on its non-target items, as close to zero as possible. The following part of the syntax which defines the non-target items for this latent variable as close as possible to zero is "av1-av6 0 av9 0 av11-av15 0 av17-av18 0 av20-av21 0 av24-av31 0 ap1-ap31 0(*1);". So, the part of the model "Vrelatedness BY av8 av10 av7 av16 av19 av22 av23 av1-av6 0 av9 0 av11-av15 0 av17-av18 0 av20-av21 0 av24-av31 0 ap1-ap31 0(*1);" defines the target items and the non-target 
Table 2. Syntax applied in software mplus 7.0 to estimate the parameters of the exploratory structural equation modeling

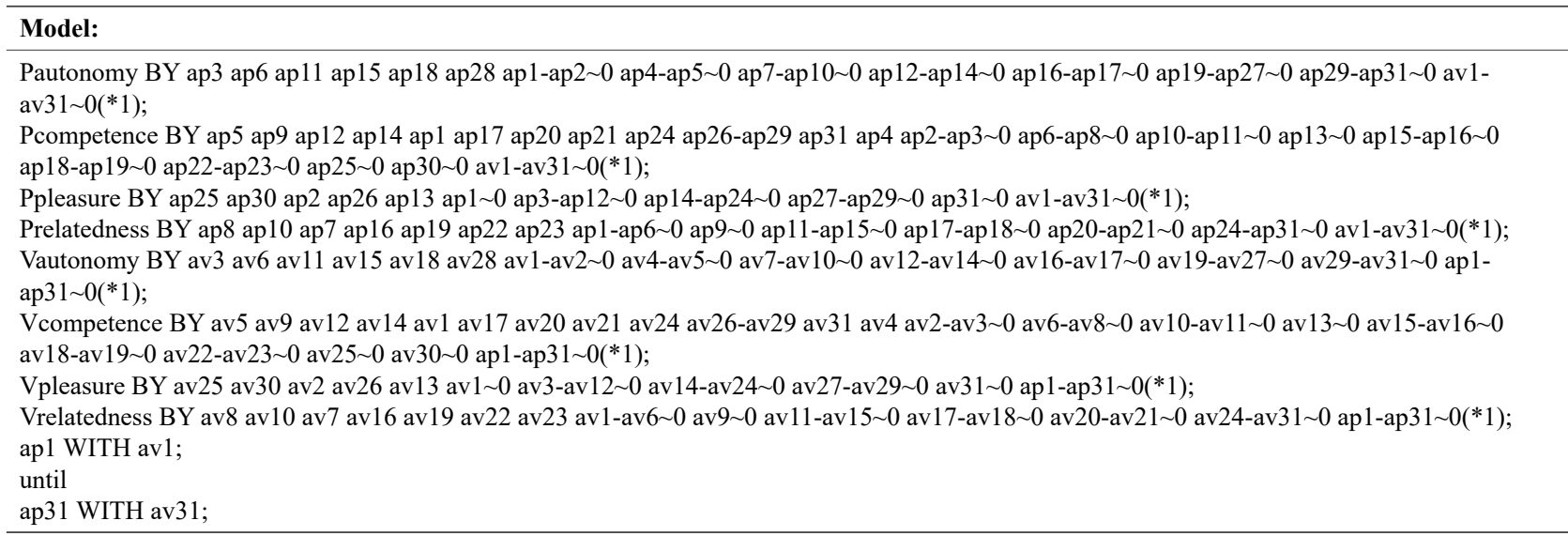

items for relatedness value, and the symbol $\sim 0$ represents as close as possible to zero. All items from the same testlet (statement) were permitted to correlate. For example, item 1 of value and item 1 of perception come from the same statement, the statement one. So, the syntax "ap1 WITH av1;" determines that these two items can correlate. The same happens for items 2, 3, and so on.

We applied the comparative fit index (CFI), the TuckerLewis index (TLI), and the root main square error of approximation (RMSEA) for inspecting the model data fit. A good data fit implies values equal or above 0.95 in CFI and TLI, as well a value below .06 in RMSEA. An unacceptable data fit is indicated by values below 0.90 in CFI and TLI, as well as equal or above .10 in RMSEA (Byrne, 2001). We applied Cronbach's alpha to estimate the reliability of the factors (Cronbach \& Meehl, 1955).

\section{RESULTS AND DISCUSSION}

Remembering, the analyzed model presupposes the presence of eight factors, which are: (1) competence perception, (2) competence value, (3) relatedness perception, (4) relatedness value, (5) autonomy perception, (6) autonomy value, (7) immediate pleasure perception, and (8) immediate pleasure value. Each of these factors possess specific target items that are loaded by them. However, the factors load on its non-target items as close as possible to zero. For example, the relatedness perception loads on items ap25, ap30, ap2, ap26, and ap13, but the other items are permitted to be loaded by this factor, as close as possible to zero, as showed in the method section.

Because the questionnaire has 31 statements and all of them generate an answer for the student's occurrence perception about each statement, and generate an answer for the student's value about each statement, there is a direct relationship between the answers of value and perception in each statement. The literature names this direct relation as a testlet (Tao, 2008). In other words, a testlet occurs when some items have the same statement and are correlated just because they come from this statement. As the SAQ has 31 statements, there are 31 testlets indicating 31 relationships between the items of value and the items of perception from a same statement. Furthermore, the model assumes that the item 1 of value and the item 1 of occurrence perception are correlated, because they come from the same statement one. The same happens for the item 2 of value and the item 2 of occurrence perception, and so on. Hence, all testlets' pairs of items (value and perception) are assumed to be correlated.

The model presented a good data fit $\left(\chi^{2}[1392]=1785.515\right.$; $\mathrm{CFI}=0.978$; TLI $=0.970$; $\mathrm{RMSEA}=.020$, with a $90 \%$ of confidence interval ranging between .017 and .023). Despite the analysis having involved all the eight factors and the 62 items, Table 3 shows only the loadings of the four perception factors on its target and non-target items, aiming at facilitating the description of them. Table 4 presents the four value factors and its loadings on target and non-target items. Describing the loadings, we will focus on items that apparently did not work well as markers of the factors, despite the reader can see through Table 3 and Table 4 the target items that were well loaded by the factors of the model. The target items of each factor are in bold grey (Table 3 and Table 4).

The competence perception loaded on four target items below .30. Item ap4 ("I work with people which think differently from me.") had a loading of .08, item ap20 ("I get upset when I receive a bad grade".) showed a loading of .09 , item ap27 ("I always choose a colleague to participate with me in the school group activities in relation to his or her competency.") had a loading of .27, and item ap29 ("I search for additional school tasks when I need extra points in order to pass to the next school year.") showed a loading of .22. Analyzing these items, ap4 was best loaded by autonomy perception (.23). Item ap20 was best loaded by competence 
Table 3. Students' Occurrence Perception Factors and Loadings on the 62 items

\begin{tabular}{|c|c|c|c|c|c|c|c|c|c|}
\hline items & PA & PC & PP & PR & items & PA & PC & PP & PR \\
\hline ap1 & .07 & .53 & .18 & .12 & av1 & .09 & .22 & .11 & -.05 \\
\hline ap2 & .06 & .09 & .12 & .26 & av2 & .10 & .09 & -.01 & .02 \\
\hline ap3 & .44 & .19 & .14 & .12 & av3 & .34 & -.06 & .09 & -.09 \\
\hline ap4 & .23 & .08 & -.01 & -.09 & av4 & .11 & .14 & -.02 & -.20 \\
\hline ap5 & .03 & .50 & -.06 & -.02 & av5 & -.05 & .16 & -.03 & -.13 \\
\hline ap6 & .54 & .11 & -.08 & .06 & av6 & .24 & -.06 & .00 & -.10 \\
\hline ap7 & .19 & .04 & .14 & .07 & av7 & .04 & .02 & .10 & -.06 \\
\hline ap8 & -.01 & .23 & .17 & .38 & av8 & -.18 & .07 & .07 & .08 \\
\hline ap9 & .25 & .30 & .05 & .10 & av9 & .05 & .03 & .05 & -.08 \\
\hline ap10 & .14 & .23 & .06 & .51 & av10 & -.02 & .03 & -.06 & .27 \\
\hline ap11 & .49 & .14 & .02 & -.03 & av11 & .24 & -.08 & .07 & -.08 \\
\hline ap12 & -.16 & .62 & .01 & .02 & av12 & -.29 & .15 & .05 & .02 \\
\hline ap13 & .23 & .25 & .25 & -.08 & av13 & .13 & .02 & .15 & -.13 \\
\hline ap14 & .05 & .62 & -.23 & -.08 & av14 & -.08 & .13 & -.12 & -.09 \\
\hline ap15 & .46 & .24 & -.01 & .20 & av15 & .24 & -.09 & -.02 & -.10 \\
\hline ap16 & .10 & .02 & .09 & .62 & av16 & -.08 & -.01 & -.12 & .27 \\
\hline ap17 & -.08 & .62 & -.09 & .09 & av17 & -.12 & .15 & .04 & .13 \\
\hline ap18 & .42 & .19 & .08 & .19 & av18 & .20 & .01 & -.15 & .04 \\
\hline ap19 & .09 & -.09 & .03 & .49 & av19 & -.05 & -.19 & -.15 & .36 \\
\hline ap20 & .16 & .09 & -.08 & .16 & av20 & .09 & .00 & -.05 & .06 \\
\hline ap21 & .18 & .51 & -.19 & -.09 & av21 & .06 & .01 & -.05 & -.03 \\
\hline ap22 & .00 & .12 & .13 & -.33 & av22 & .01 & .02 & .05 & -.27 \\
\hline ap23 & .08 & .06 & -.08 & .49 & av23 & .04 & -.07 & -.13 & .42 \\
\hline ap24 & .19 & .37 & -.21 & .09 & av24 & .09 & -.01 & -.10 & .05 \\
\hline ap25 & .01 & -.24 & .67 & .11 & av25 & -.05 & .05 & .13 & -.10 \\
\hline ap26 & -.02 & .59 & -.36 & .03 & av26 & -.06 & .04 & -.05 & .02 \\
\hline ap27 & .12 & .27 & .05 & .02 & av27 & -.04 & .15 & .04 & -.03 \\
\hline ap28 & .32 & .36 & -.04 & -.03 & av28 & .19 & -.04 & .04 & -.08 \\
\hline ap29 & .26 & .22 & -.07 & .03 & av29 & .13 & -.04 & -.06 & .05 \\
\hline ap30 & -.04 & -.18 & .61 & .09 & av30 & -.05 & .02 & .17 & -.05 \\
\hline ap31 & -.01 & .47 & .23 & .04 & av31 & -.09 & .08 & .22 & -.06 \\
\hline
\end{tabular}

Legend: PA-occurrence perception of autonomy; $P C=$ occurrence perception of competence; PP = occurrence perception of immediate pleasure; PR = occurrence perception of relatedness; ap = items of occurrence perception; av = items of value.

value (.25), indicating that the students answered this item not principally by their perception of occurrence, but by their value about it. Despite item ap27 having a low loading by the competence perception, it was its best loading. Lastly, item ap29 was best loaded by autonomy perception. None of the non-target items was loaded equal or above .30 by competence perception.

The competence value loaded three target items below .30. They are the items av4, av5 and av20 (respectively .04, .26 , and .24). Despite having shown low loadings, the items av5 ("I search for activities which I am not so good at, aiming at working on my difficulties") and av20 (already presented) were highest loaded by this factor. Four non-target items have been loaded equal or above .30 by competence value. They are the items av6 ("I take the responsibility for my choices"), av13 ("I choose people for school group tasks based on common interests, even if they are not my friends"), av15 ("I have clarity about what I want"), and av18 ("I choose what I consider the best for me"), indicating that some personal interest and autonomy statements have some direct relation to the competence values of the students.

The autonomy perception loaded on all target items with at least a value of .32. Only one non-target item was loaded equal or above .30 by this factor, which is item av3. The autonomy value loaded many target items with values below .30, considering that this factor has six target items and four of them had low loadings. These are the items av3 (.28), av15 (.24), av18 (.20) and av28 (.05). Even the other two target items with loadings equal or above .30 presented relatively low loadings (item av6 showed a loading of 36 and item av11 had a loading of .37). About the non-target items, the items ap6 and ap11, which are target items of 
Table 4. Students' Value Factors and Loadings on the 62 items

\begin{tabular}{|c|c|c|c|c|c|c|c|c|c|}
\hline items & VA & VC & VP & VR & items & VA & VC & VP & VR \\
\hline ap1 & -.13 & -.05 & -.01 & .01 & av1 & -.02 & .30 & .05 & .12 \\
\hline ap2 & .07 & -.08 & .13 & -.08 & av2 & .12 & -.18 & .34 & .30 \\
\hline ap3 & -.08 & .04 & -.06 & .07 & av3 & .28 & .19 & -.04 & .24 \\
\hline ap4 & -.10 & -.02 & -.07 & .13 & av4 & .05 & .04 & -.10 & .23 \\
\hline ap5 & -.10 & -.06 & -.01 & .10 & av5 & .23 & .26 & -.12 & .29 \\
\hline ap6 & .31 & -.14 & -.05 & .06 & av6 & .36 & .35 & -.16 & .20 \\
\hline ap7 & -.15 & -.01 & -.14 & .27 & av7 & -.03 & .11 & .03 & .51 \\
\hline ap8 & -.01 & .04 & -.06 & .09 & av8 & .14 & .21 & .01 & .50 \\
\hline ap9 & .17 & -.06 & -.01 & -.01 & av9 & .37 & .34 & -.12 & .15 \\
\hline ap10 & -.07 & -.03 & -.08 & .23 & av10 & .13 & .24 & .01 & .52 \\
\hline ap11 & .33 & -.13 & -.03 & -.05 & av11 & .37 & .23 & -.01 & .19 \\
\hline ap12 & .22 & .00 & -.06 & -.09 & av12 & .31 & .57 & -.12 & .07 \\
\hline ap13 & .00 & .01 & .18 & .04 & av13 & .04 & .31 & .23 & .08 \\
\hline ap14 & .01 & .13 & .00 & -.06 & av14 & .29 & .61 & -.11 & .08 \\
\hline ap15 & .01 & -.03 & .02 & -.16 & av15 & .24 & .41 & .00 & .07 \\
\hline ap16 & -.06 & -.06 & -.05 & .08 & av16 & .11 & .01 & .32 & .35 \\
\hline ap17 & .04 & .23 & .07 & -.31 & av17 & .06 & .65 & .09 & -.10 \\
\hline ap18 & .18 & .06 & .11 & -.18 & av18 & .20 & .31 & .27 & .07 \\
\hline ap19 & -.02 & -.06 & .02 & .29 & av19 & .10 & .04 & .17 & .54 \\
\hline ap20 & -.05 & .25 & .00 & -.10 & av20 & .01 & .24 & .10 & .00 \\
\hline ap21 & -.11 & .06 & .02 & .13 & av21 & -.04 & .69 & -.20 & .10 \\
\hline ap22 & .11 & .04 & .02 & -.15 & av22 & .01 & .20 & .35 & .00 \\
\hline ap23 & .04 & .00 & .00 & .18 & av23 & .11 & .28 & .07 & .16 \\
\hline ap24 & .03 & .15 & -.02 & -.01 & av24 & .08 & .71 & -.03 & -.06 \\
\hline ap25 & .16 & .02 & .16 & -.06 & av25 & .10 & -.23 & .73 & .13 \\
\hline ap26 & -.03 & -.01 & .05 & .07 & av26 & .02 & .69 & -.24 & .03 \\
\hline ap27 & -.16 & .00 & .16 & -.02 & av27 & -.18 & .39 & .26 & -.07 \\
\hline ap28 & .00 & .12 & .05 & -.09 & av28 & .05 & .62 & .02 & .05 \\
\hline ap29 & -.26 & .09 & .04 & .06 & av29 & -.15 & .54 & .00 & .01 \\
\hline ap30 & -.10 & .08 & .15 & -.11 & av30 & -.14 & -.05 & .68 & .09 \\
\hline ap31 & -.11 & .08 & -.03 & .13 & av31 & -.06 & .55 & -.08 & .22 \\
\hline
\end{tabular}

Legend: VA-value of autonomy; VC = value of competence; VP=value of immediate pleasure; VR = value of relatedness; ap = items of occurrence perception; $a v=$ items of value.

autonomy perception, have been loaded above .30 by autonomy value. Beyond ap 6 and ap 11 items, autonomy value loaded equal or above .30 on items av9 ("I am capable of thinking in other ways to solve a problem when the first try does not work out") and av12 ("I have good grades in the school evaluations"), which represent academic selfefficacy aspects.

The immediate pleasure perception loaded bellow 30 on target items ap2 ("The most part of my time I do things that I like"), and ap13 ("I choose people for school group tasks based on common interests, even if they are not my friends"). None of the non-target items were loaded equal or above .30 by this factor. The immediate pleasure value loaded below . 30 on target items av13 (already showed) and av26 ("Even when I do not like a certain school activity, I do it with excellence"), which the last is an inverse, or negative, marker of this factor. This factor showed loadings a little above .30 on the following non-target items: av16 ("I see school as a place to make friends"), and av22 ("I prefer working alone than working in groups").

The relatedness perception loaded on only one target item below .30, which is the item ap7 ("I make friends through assignments or group meetings"). Two value items (av19 and av23) were loaded equal or above .30 by this factor, indicating that the perception of occurrence had a direct influence in these two value items of relatedness. The relatedness value showed two loadings below .30 on target items av22 ("I prefer working alone than working in groups") and av23 ("I have school friends which I should always count on"). Two non-target items, av2 and ap17, have been loaded around .30 by this factor. Item ap 17 ("I aim at being the best student of my class") was negatively loaded 
by it, indicating that the need of relatedness is partially non-compatible with the desire of being the best student, which makes theoretical sense. The load of the relatedness value on item av2 ("The most part of my time I do things that I like") may indicate a connection, despite being small, between being related with others and feeling pleasure and doing funny activities.

For computing the Cronbach's alpha of the eight factors, we have selected preferentially all target items and only nontarget items with loadings equal or above 30 . We eliminated for these calculations the items av 22 and av 17 for relatedness value, and only have included the items av 25 and av 30 for immediate pleasure value, as well only included the items ap25 and ap30 for immediate pleasure perception. All other factors have its alpha calculated including all target items and non-target with loadings equal or above .30 . Table 5 shows in diagonal (italicized) the alphas. The lowest alpha occurred in pleasure perception (.634) and the highest alpha was present in competence value (.792). Despite of .70 values or above should be considered better, .60 values are reasonable, which permit us to infer that all factors achieved the minimum cut-off value for reliability.

The correlations between the items of the testlets presented a considerable mean of .557 (standard deviation of .118). The lowest correlation was in ap12 and av12 (.15), and the highest correlation occurred in ap7 and av7 (.74). The correlations of the testlets have to be considered when interpreting the loadings of the factors on its target items. If we did not evaluate a solution with all the 62 items, incorporating the testlets, a considerable part of its correlations would have been transferred to the factors and its target items. Hence, the loadings were attenuated because of the testlets' presence.

Synthetizing, in general all factors were well identified because they loaded predominantly on the target items, as well on few non-target items. Besides, the non-target items loaded by the factors were not incompatible and showed theoretical fit. Table 5 shows the correlation matrix of the eight factors. Some correlations are apparently more relevant to understand possible connections among the factors. We are considering in the results principally the correlations around .30 or above.

The competence perception showed a relevant correlation with competence value and autonomy perception. The competence value showed a connection with autonomy perception, similar to competence perception, as well correlated with the values of autonomy and relatedness.

The autonomy perception related with competence (perception and value, as already pointed), as well with relatedness value. The perception and value of autonomy showed a very low correlation (.068), indicating that the student's perception of its occurrence was not related with their values of autonomy. The autonomy value related with competence value, as already said.

The relatedness factors showed a weak correlation between them (.131), indicating, as occurred in autonomy, that the students' occurrence perception of relatedness is almost orthogonal, or independent from their value about relatedness. The relatedness perception did not show any considerable correlation with other factors, and its biggest correlation has been with immediate pleasure value. The relatedness value showed connections with competence value and autonomy perception.

The immediate pleasure perception showed low correlations with all other factors, and its biggest value was with immediate pleasure value. The same happened with immediate pleasure value. The two immediate pleasure factors showed negative correlations or almost orthogonal relations with the other factors. Curiously, immediate pleasure perception correlated positively with relatedness value, and immediate pleasure value correlated positively with relatedness perception.

Comparing our results with the literature of selfdetermination theory, we need to inform again that the SAQ is the first instrument that separates values and occurrence perceptions, as well integrates the immediate pleasure together with the three basic needs. So, the relationships between immediate pleasure and the three basic needs, in terms of values and occurrence perceptions is a peculiar result of SAQ, which is reported on this manuscript. The correlations of the values in relation to the occurrence

Table 5. Correlations Matrix of the Eight Factors Model

\begin{tabular}{|c|c|c|c|c|c|c|c|c|}
\hline & PC & PA & PR & PP & VC & VA & VR & $\mathbf{V P}$ \\
\hline PC & .779 & & & & & & & \\
\hline PA & .331 & .700 & & & & & & \\
\hline PR & .101 & .079 & .700 & & & & & \\
\hline PP & -.020 & -.018 & -.085 & .634 & & & & \\
\hline $\mathrm{VC}$ & .281 & .313 & .015 & -.111 & .792 & & & \\
\hline VA & .096 & .068 & .043 & -.099 & .343 & .779 & & \\
\hline VR & .082 & .298 & .131 & .095 & .325 & .202 & .661 & \\
\hline VP & .007 & .014 & .158 & .182 & -.039 & -.106 & -.079 & .670 \\
\hline
\end{tabular}

Legend: $P C=$ occurrence perception of competence; $P A$-occurrence perception of autonomy; $P R=$ occurrence perception of relatedness; $P P=$ occurrence perception of immediate pleasure; $V C=$ value of competence; $V A-$ value of autonomy; $V R=$ value of relatedness; $V P=$ value of immediate pleasure. 
perceptions follow the same idea. They are original and peculiar of our work. Despite of these specificities, observing the results of previous studies (synthetized in Chen et al., 2015), we found a similar result which is the evidence that autonomy and competence are more correlated with themselves in comparison to relatedness. However, our results and the previous evidences should be compared with some cautious, since the correlations between autonomy and competency found in those studies (synthetized in Chen et al., 2015) are all sustained through data from the traditional instruments of the basic needs which focus on satisfaction or frustration. Those instruments do not assess how much the basic needs are perceived by people as occurring in terms of frequency. These differences in the assessment are relevant and should be considered when trying to compare our results with the previous studies. Until now, a unique study (Chen et al., 2015) has considered values as variables to be analyzed together with the satisfaction or frustration of the basic needs. However, that study operationalized the values as the desire of change about the needs (study 2 of Chen et al., 2015), or showed serious problems in the reliability of the instruments (study 1 of Chen et al., 2015), precluding us to compare our results with that study.

\section{CONCLUSION}

In spite of being acknowledged as a relevant theory in the world scenario, self-determination theory is growing only recently in Brazil. Guimarães and Boruchovitch (2004) pointed in 2004 that the theory was not widely disseminated in Brazil. However, the tendency is of increasing, and the School Aspirations Questionnaire (SAQ) should be seen as pertaining in this context.

The good data fit found by the model of eight correlated factors permit us to say that the SAQ was capable of not only measuring the students' occurrence perception of autonomy, competence, relatedness, and immediate pleasure, but it was also capable of measuring the students' value of autonomy, competence, relatedness, and immediate pleasure. This is the first instrument that separates the students' values and the students' occurrence perception about the basic needs of the self-determination theory, aggregating a four domain, which is immediate pleasure. About the correlations among the factors, we found that only the competence domain showed a relevant correlation between value and occurrence perception of the students, which is intriguing. The other domains presented a weak positive correlation, indicating an almost independent relation between value and perception of occurrence. We do not know if this result is a particularity of our sample or is a more general pattern existent in middle school students and high school students. Because we have only concentrated in the structural validity of SAQ, new studies are necessary to investigate this question. As we commented previously, the testlets showed that the items from the same statement are related, so the loadings among the factors and its target items are attenuated by the testlets. The correlations found in testlets are much common and pointed by literature (Tao, 2008), and despite the advantage of possessing a statement which serves as a common platform for different items (in our case, values and perceptions), researchers should interpret loadings with some cautious and should be lessen rigorous with lower loadings because of the attenuation situation provoked by the testlet presence.
The SAQ should be considered an advance for the field, because it can inform researchers the level of the basic needs perceived by the students in the school context, as well as how they value each of these needs. Different profiles of students can be investigated through the questionnaire. For example, researchers should verify if there are students with high values in all three needs which perceive a low occurrence of these needs in their school life, showing a contrasting aspect between their values and their perceptions. Researchers should observe if there are students who value poorly certain basic needs in the school context, aiming at understanding if some profiles tend to be associated with low quality of life, well-being, functional adjustment, or academic achievement. It is possible that some tensions or dissonances between perceptions and values should be associated with dysfunctional adjustment or low quality of life in the school context.

Self-determination, as any scientific theory, possesses important questions to be invested and investigated. Vallerand, Pellletier and Koestner (2008) indicate the need for more research about the implications evolved when one or more basic needs are not promoted satisfactorily in the development of the individual. They argue about the necessity of that research agenda, which brings the perspective of study the imbalances among the basic needs of self-determination. As Vallerand et al. (2008) suggest, it is possible that the eudemonic life style, which focuses on intrinsic values and motivations, should favor a more equilibrium among the basic needs and support a stronger and stable well-being and quality of life. As commented previously, the SAQ should work on these questions, according as it should focus on how the connections between values and perceptions of the basic needs are capable of producing different forms of personal or group adaptations.

We understand that the theory does not focus on individual differences from the three basic needs, postulating that all people possess a strong mobilization for them (Chen et al., 2015). However, the more a theory defies its own postulations, the better. After all, what makes a theory 
stronger is its capacity to maintain their assumptions after the scrutiny of the empirical plain. The more a theory defends itself about that scrutiny, the more the theory undermines its own forces and credibility. Hence, the evaluation of individual differences about the basic needs, as well the investigation of different profiles that generate multiple types of adaptations and mal-adjustments should be an advance for the self-determination theory.

We expect to study the connections among the students' perceptions and values of the basic needs in relation with academic achievement and quality of life in middle and high school. As we focused this paper on structural validity, a next study investigating external validity (convergent validity or predictive validity) will improve the validity of the School Aspirations Questionnaire. Besides, we hope to amplify the sample, incorporating students of other regions of Brazil, as well as socio-economic statuses and public schools, which will permit us to investigate the invariance of the SAQ. As reported, the results of this manuscript comes from students' data of just a school.

\section{REFERENCES}

Black, A. E., \& Deci, E. L. (2000). The effects of instructors autonomy support and students' autonomous motivation on learning organic chemistry: A self-determination theory perspective. Science Education, 84, 740-756. doi: $10.1002 / 1098-237 \mathrm{X}(200011) 84: 6<740:$ :AIDSCE4>3.0.CO;2-3

Byrne, B. M. (2001). Structural equation modeling with AMOS: Basic concepts, applications, and programming. Mahwah: Lawrence Erlbaum Associates.

Chen, B., Vansteenkiste, M., Beyers, W., Boone, L., Deci, E. L., Van der Kaap-Deeder, J., ... Verstuyf, J. (2015). Basic psychological need satisfaction, need frustration, and need strength across four cultures. Motivation and Emotion, 39(2), 216-236. doi: 10.1007/s11031-014-9450-1

Cronbach, L. J., \& Meehl, P. E. (1955). Construct validity in psychological tests. Psychological Bulletin, 52(4), 281-302.

Deci, E. L., \& Ryan, R. M. (1985). Intrinsic motivation and selfdetermination in human behavior. New York: Plenum.

Deci, E. L., \& Ryan, R. M. (2000). The "what" and "why" of goal pursuits: Human needs and the self-determination of behavior. Psychological Inquiry, 11, 227-268. doi: 10.1207/ S15327965PLI1104 01

Deci, E. L., \& Ryan, R. M. (2016). Optimizing students' motivation in the era of testing and pressure: A self-determination theory perspective. In L. W. Chia, J. W. C. Keng, \& R. M. Ryan (Eds.), Building autonomous learners: Perspectives from research and practice using self-determination theory (pp. 9-29). London: Springer.

Early, D. M., Rogge, R. D., \& Deci, E. L. (2014). Engagement, alignment, and rigor as vital signs of high-quality instruction: A classroom visit protocol for instructional improvement and research. The High School Journal, 97(4), 219-239. doi: 101353/hsj.2014.0008

Gomes, M. A. M., \& Boruchovitch, E. (2015). Escala de motivação para a leitura do ensino fundamental: Construção e validação. Psicologia: Reflexão e Crítica, 28(1), 68-76. doi: 10.1590/1678-7153.201528108

Guimarães, S. E. R, \& Boruchovitch, E. (2004). O estilo motivacional do professor e a motivação intrínseca dos estudantes: Uma perspectiva da teoria da autodeterminação. Psicologia: Reflexão e Crítica, 17(2), 143-150. doi: 10.1590/ S0102-79722004000200002
Hagger, M. S., \& Chatzisarantis, N. L. D. (2016) The trans-contextual model of autonomous motivation in education: Conceptual and empirical issues and meta-analysis. Review of Educational Research, 86(2), 360-407. doi: 10.3102/0034654315585005

Marsh, H. W., Morin, A. J. S., Parker, P. D., \& Kaur, G. (2014). Exploratory structural equation modeling: An integration of the best features of exploratory and confirmatory factor analysis. Annual Review of Clinical Psychology, 10, 85-110. doi: 10.1146/annurev-clinpsy-032813-153700

Muthén, L. K., \& Muthén, B.O. (1998-2014). Mplus users's guide. Los Angeles: Muthén \& Muthén.

Ryan, R. M., \& Connell, J. P. (1989). Perceived locus of causality and internalization: Examining reasons for acting in two domains. Journal of Personality and Social Psychology, 57(5), 749-761. doi: 10.1037/0022-3514.57.5.749

Silva, M. N., Marques, M. M., \& Teixeira, P. J. (2014). Testing theory in practice: The example of self-determination theorybased interventions. The European Health Psychologist, 16(5), 171-180. doi: 10.1186/1479-5868-9-20.

Tao, W. (2008). Using the score-based testlet method to handle local item dependence (Doctoral dissertation). Lynch School of Education, Boston College, Department of Educational Research, Measurement, and Evaluation.

Vallerand, R. J., Pelletier, L. G., Koestner, R. (2008). Reflections on self-determination theory. Canadian Psychology, 49(3), 257-262. doi: http://dx.doi.org/10.1037/a0012804

Walker, C. O., Winn, T. D., \& Lutjens, R. M. (2012). Examining relationships between academic and social achievement goals and routes to happiness. Education Research International, 2012, 1-7, doi: 10.1155/2012/643438. 